\title{
Enteric Nervous System Impairment in Gastroschisis
}

\author{
Frederic Auber ${ }^{1}$ Enrico Danzer ${ }^{2}$ Marie-Emmanuelle Noché-Monnery ${ }^{1}$ Sabine Sarnacki ${ }^{3}$ \\ Germain Trugnan ${ }^{4}$ Sabah Boudjemaa ${ }^{5}$ Georges Audry ${ }^{1,6}$
}

${ }^{1}$ Department of Pediatric Surgery, Hospital Armand Trousseau/
Hopitaux Universitaire Est Parisien/AP-HP, Paris, France
${ }^{2}$ The Center for Fetal Research, Children's Hospital of Philadelphia,
Philadelphia, Pennsylvania, United States
${ }^{3}$ Department of Pediatric Surgery, Hospital Necker Enfants-Malades,
Paris, France
${ }^{4}$ Laboratoire ER7, Faculté de Médecine Pierre et Marie Curie, site Saint-
Antoine, Paris, France
${ }^{5}$ Service d'Anatomie et Cytologie Pathologiques, AP-HP/Hospital
Armand Trousseau/Hopitaux Universitaire Est Parisien, Paris, France
6 Faculté de Médecine, Université Pierre et Marie Curie, Paris, France

Address for correspondence and reprint requests Frederic Auber, MD, PhD, Department of Pediatric Surgery, Hospital Armand Trousseau/ Hopitaux Universitaire Est Parisien/AP-HP, 26 avenue du Docteur Arnold Netter, Paris 75012, France (e-mail: frederic.auber@trs.aphp.fr).

Eur J Pediatr Surg 2013;23:29-38.

\begin{abstract}
Keywords

- gastroschisis

- enteric nervous system

- interstitial cells of Cajal

- experimental models

Introduction After surgical repair of gastroschisis, most neonates exhibit severe intestinal dysmotility. We hypothesized that impaired development of the enteric nervous system or interstitial cells of Cajal (ICC) network contributes to impaired intestinal motility in gastroschisis. We evaluated this hypothesis in a rat model of gastroschisis.

Material and Methods Gastroschisis was created surgically in rat fetuses on gestational day 18, under general anesthesia, and small bowel was harvested on day 22 . Intestinal weight-to-length (IW/L) ratio, and small-bowel wall thickness were assessed. Specimens were processed for hematoxylin-eosin staining or immunohistochemistry with specific markers for neuronal cells (Hoxb5), glial cells (GFAP, S100), and ICCs (c-kit). Myenteric plexus maturation was assessed morphologically and compared with sham and control fetuses. Stage of development of the myenteric plexus was graded from 1 (mature) to 3 (very immature) comparatively with specimens from E16 to E22 control fetuses.

Results Compared with sham-operated or control fetuses, gastroschisis was associated with increases in mean intestinal weight/intestinal length (IW/L) ratio, and mean thicknesses of the total, muscular, and submucosal layers of the small-bowel wall. The myenteric plexus were present in the small bowel from fetuses with gastroschisis, however all exhibited abnormal myenteric plexus maturation. Thus, of the gastroschisis fetuses, $55 \%$ had an aspect similar to the immature myenteric plexus of E19-E20 fetuses and $45 \%$ to the very immature mesenteric plexus observed in E16-E18 fetuses. When compared with sham and control groups, ICCs were less abundant in eviscerated small bowel in the gastroschisis group and often exhibited weak c-kit staining or an abnormally round shape without branches. Hoxb5, a marker for enteric neuroblasts and neuronal precursors, was expressed similarly in myenteric plexuses in all groups. S100 or GFAP
\end{abstract}

received

May 18, 2012

accepted after revision

July 18, 2012

published online

October 25, 2012 (c) 2013 Georg Thieme Verlag KG

Stuttgart · New York
DOI http://dx.doi.org/ 10.1055/s-0032-1326955. ISSN 0939-7248. 
staining showed the presence of glial cells within the myenteric plexuses in all groups. The $\mathbf{S 1 0 0}$ expression level was similar in all groups. In contrast, the GFAP expression level was higher in the gastroschisis group than in the sham and control groups.

Conclusion Our results suggest that delayed maturation of the enteric nervous system combined with impaired ICC network development may largely explain the intestinal dysmotility seen in neonates with gastroschisis.

\section{Introduction}

Gastroschisis is a congenital abdominal wall defect that poses several challenges to surgeons and pediatricians. In this condition, the small bowel is herniated through the abdominal wall defect and is directly exposed to amniotic fluid in utero. ${ }^{1,2}$ After birth, surgery and supportive care must be provided promptly. Following surgical repair, many patients exhibit severe intestinal dysmotility that requires prolonged parenteral nutrition. ${ }^{1-4}$ These neonates are subsequently at risk for sepsis and parenteral nutrition-induced liver disease. Although the mortality rate of live-born gastroschisis patients has decreased over time and is now less than $10 \%$, the morbidity rate remains high as a result of prolonged intestinal dysmotility, associated intestinal atresia, short bowel syndrome, and iatrogenic adverse events. ${ }^{1-4}$ The initiation and regulation of small-bowel motility require the normal function of several cell populations. The enteric nervous system is a neuronal network located all along the gut that regulates intestinal peristalsis and secretion. ${ }^{5}$ Within the small-bowel wall, the enteric nervous system has two components, the submucosal plexus and the myenteric plexus, both made up of neurons and glial cells derived from the neural crest. ${ }^{5}$ The interstitial cells of Cajal (ICC) are cells derived from the mesoderm and serve as electrical pacemakers in the gut wall, generating and propagating the electrical signal to the smooth muscle. ${ }^{6}$ The smooth muscle cells are the final effector cells responsible for small-bowel motility.

Given that severe intestinal dysmotility is a prominent feature in neonates with gastroschisis, we investigated the hypothesis that gastroschisis may be associated with impairment of the enteric nervous system or ICC network. To this end, we used a previously established rat model of gastroschisis ${ }^{7}$ and assessed the phenotype of the enteric nervous system and ICC network in the eviscerated small bowel.

\section{Methods}

\section{Rat Model of Gastroschisis}

Animal care and use complied with French regulations regarding experiments on animals. Time-dated pregnant rats were purchased and two were housed to a cage on a 12-hour dark cycle/12-hour light cycle with food and water ad libitum in the laboratory animal facility of the INSERM Research Center (Saint-Antoine Hospital, Paris, France).

Gastroschisis was created surgically in rat fetuses as previously described by Correia-Pinto et al. ${ }^{7}$ Briefly, pregnant rats were anesthetized on gestational day 18 using intramuscular ketamine (Panpharma, France) and chlorpromazine (Sanofi-Aventis, France), with additional inhalation of sevoflurane (Abbott, UK) if needed. The uterus was exposed through a midline laparotomy and opened in the middle of a 7/0 polydioxanone (Ethicon, Belgium) purse string. Then, under surgical microscope visualization, an incision was performed in the right or left lower abdominal quadrant and gentle pressure was applied to the fetal abdomen to extrude bowel loops through the incision. The other fetuses received either only manipulation (sham-operated group) or no intervention (control group). Warm saline solution was infused into the amniotic cavity and the purse string was closed. The laparotomy was closed in two layers with continuous absorbable sutures. To compensate for the high mortality rate expected after gastroschisis creation, we performed the gastroschisis and sham procedures in 80 and 30 fetuses, respectively. For each dam, we created a diagram of the uterus showing the position and group (gastroschisis, sham, or control) of each fetus.

\section{Harvesting and Macroscopic Assessment of Small Bowel}

The entire small bowel of each rat fetus in each of the three groups (gastroschisis, sham, and control) was harvested on day 22 of gestation under general anesthesia. Fetuses that died in utero or exhibited necrosis of the eviscerated small bowel were excluded from the analysis. Eviscerated and noneviscerated small bowel specimens were assessed and processed separately. Presence of a fibrous coating or peel on the eviscerated small bowel in the fetuses with gastroschisis was assessed macroscopically. The length of the small bowel was measured in millimeters and the weight in $\mathrm{mg}$; these values were used to compute the ratio of intestinal weight over intestinal length (IW/L). In some instances extensive peel formation with severe edema and retraction precluded measurement of small-bowel length. Therefore, we defined a weight score combining intestinal weight/intestinal length (IW/L) values and macroscopic assessment of peel formation. IW/L was converted to a weight score as follows: $1, \mathrm{IW} / \mathrm{L}=0$ to $15 \mathrm{mg} / \mathrm{cm} ; 2, I W / L=15$ to $30 \mathrm{mg} / \mathrm{cm} ; 3, I W / L=30$ to $45 \mathrm{mg} / \mathrm{cm}$; and $4, \mathrm{IW} / \mathrm{L}>45 \mathrm{mg} / \mathrm{cm}$ or major peel preventing length measurement. Thus the highest possible score (4) was either $\mathrm{IW} / \mathrm{L}>45 \mathrm{mg} / \mathrm{cm}$ or major peel preventing length measurement, which allowed the evaluation of all small bowels including those with severe damage. After macroscopic assessment, a small-bowel specimen from each fetus 
was collected, fixed in $10 \%$ formalin, and embedded in paraffin.

\section{Histology and Immunohistochemistry}

Serial $5-\mu \mathrm{m}$ transversal sections, at least 50 per specimen, were obtained from the paraffin-embedded small-bowel specimens and processed for hematoxylin-eosin staining or immunohistochemistry. Each slide had an identification number with no reference to the group of the fetus, to keep assessor "blind" during analysis. Immunochemistry was performed according to the standard procedures. Briefly, paraffin sections were air-dried overnight in a $50^{\circ} \mathrm{C}$ oven, deparaffinized, and rehydrated through decreasing alcohol series down to distilled water. The sections were incubated for 30 minutes with peroxidase-blocking reagent (DakoCytomation, Glostrup, Denmark). Heat-induced epitope retrieval was performed by immersion in antigen unmasking solution (Vector Laboratories, Burlingame, California, United States) and microwave heating for all stains except $\alpha$-smooth-muscle actin. The sections were incubated with the primary antibody for 12 hours at $4^{\circ} \mathrm{C}$ then with the secondary poly-HRB conjugate antibody (Zymed Invitrogen, Carlsbad, California, United States) for 30 minutes at room temperature. The sections were rinsed in phosphate-buffered saline, and immunoreactivity was detected at room temperature by addition of DAB substrate (ref SK-4100, Vector Laboratories) for 4 minutes. Counterstaining was done with Harris hematoxylin.

The following markers were studied by immunohistochemistry ( - Table 1 ): $\alpha$-smooth-muscle actin (smooth muscle cells), GFAP (intracytoplasmic filamentous protein found in the cytoskeleton of astrocytes and enteric glial cells), S100 protein (a calcium-binding protein abundant in glial cells of the central and peripheral nervous system), Hoxb5 (enteric neuroblasts and neuronal precursors), and c-kit (ICCs). We performed a preliminary titration and crossreactivity study of the antibodies on small-bowel specimens from control rats before using them in the study.

Light Microscopy: To assess small-bowel inflammation, in addition to peel formation, the IW/L ratio, and the weight score, we used five continuous variables: total thickness of the small-bowel wall $(\mu \mathrm{m})$, thickness of the small-bowel muscular layer $(\mu \mathrm{m})$, thickness of the small-bowel submucosa $(\mu \mathrm{m})$, inner width of the small-bowel villi (chorion of the villus measured between the epithelium basement membranes on each side, in $\mu \mathrm{m}$ ), and outer width of the smallbowel villi (total width of the villus measured between the epithelium apical membranes on each side, in $\mu \mathrm{m}$ ).

These variables were evaluated by observing serial $5-\mu \mathrm{m}$ transversal sections under the light microscope, and analysis was performed on circular sections of small bowel, at least 10 per specimen. The investigator assessing the sections was "blind" to the group of the specimen. Digital images were obtained on a Leica DMR microscope (Wetzlar, Germany) equipped with a charge-coupled device camera (3CCD Color Video Camera Model DXC-950P, Sony, Japan) and recorded with Tribvn version 1.3 software (Châtillon, France). The overall structure of the small-bowel layers was assessed and morphometric variables were obtained using imageanalysis software (SigmaScan Pro 5 Aspire Software International, Ashburn, Virginia, United States). For each variable in each fetus, the mean of 10 measurements was used for the study.

The enteric nervous system was assessed by a qualitative analysis of the structure of the myenteric plexus lying between the two muscular layers of the small bowel after $\alpha$ smooth-muscle actin immunostaining and hematoxylin counterstaining. Stage of development of the myenteric plexus was graded from 1 (mature) to 3 (very immature) comparatively with specimens from E16 to E22 control fetuses (-Fig. 1). Grade 1 (mature) myenteric plexus was observed in E21-E22 control fetuses and defined as a discontinuous belt of well-defined ganglia with round cells, grade 2 (immature) was observed in E19-E20 control fetuses and defined as a discontinuous belt with ovoid cells, and grade 3 (very immature) was observed in E16-E18 control fetuses and defined as a continuous belt with ovoid or spindle-shaped cells. Expression of Hoxb5, GFAP, and S100 proteins was assessed after immunostaining and graded using a semiquantitative 3-point scale (1, weak expression; 2, medium expression; and 3, strong expression). For each marker in each fetus, at least 10 transverse sections were evaluated. Strong expression was defined as the presence of highly positive cells in all myenteric plexus. Medium expression was defined as the presence

Table 1 Antibodies used for the immunohistochemistry studies of fetal rat small-bowel specimens

\begin{tabular}{|c|c|c|c|}
\hline Labeled cell type & Antibody & Supplier and references & Dilution \\
\hline Smooth muscle cells & Mouse antihuman $\alpha$-smooth-muscle actin ${ }^{a}$ & $\begin{array}{l}\text { Dako }{ }^{\mathrm{b}} \text {, monoclonal purified, clone 1A4, } \\
\text { ref. M0851 }\end{array}$ & $1: 100$ \\
\hline Enteric neurons & Rabbit antirat Hoxb5 & $\begin{array}{l}\text { Polyclonal, generated by MV Volpe, } \\
\text { Tufts Medical Center, Boston, } \\
\text { Massachusetts, United States }\end{array}$ & $1: 100$ \\
\hline Glial cells & Rabbit anticow S100B & Dako $^{\text {b }}$, polyclonal purified, ref. Z0311 & $1: 400$ \\
\hline Glial cells & Rabbit anticow GFAPa & Dakob, polyclonal purified, ref. Z0334 & $1: 10$ \\
\hline Interstitial cells of Cajal & Rabbit antihuman C-KITa & 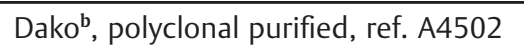 & $1: 50$ \\
\hline
\end{tabular}

${ }^{\mathrm{a} C}$ Cross-react with same rat antigen.

bDakoCytomation, Glostrup, Denmark. 

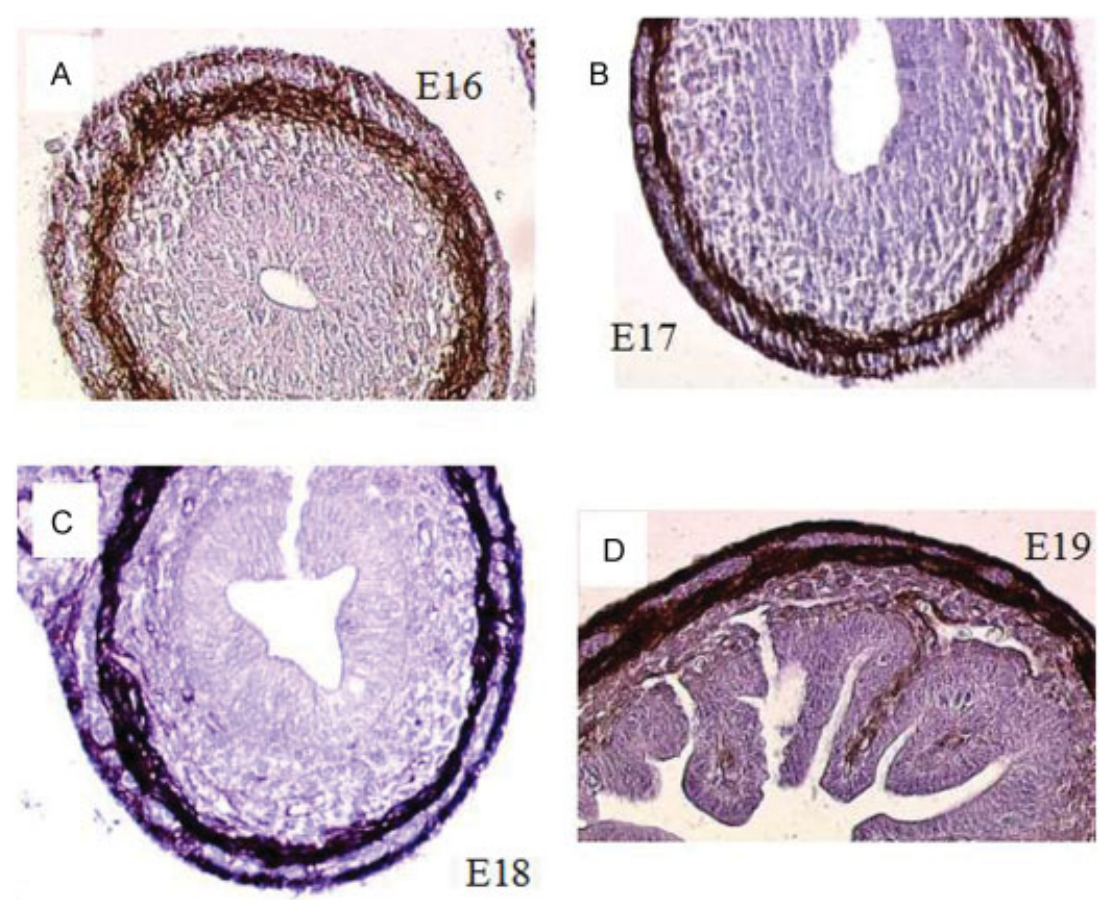

E18


Figure 1 Small bowel from control fetuses harvested from E16 to E21, magnification x10 (panel A-C) or x20 (panel D-F). Immunostaining with anti- $\alpha$-smooth-muscle actin antibody.

of highly positive cells in at least $50 \%$ of myenteric plexus per circular section. Weak expression was defined as the presence of positive cells in at least one myenteric plexus per circular section.

ICCs were evaluated using light microscopy after c-kit immunostaining and hematoxylin counterstaining. The presence and appearance of the ICC network were recorded. The ICC network was considered normal when the cells showed both strong c-kit expression and a typical spindle shape with thin branches extending around the myenteric plexus. The ICC network was considered abnormal when either criterion was absent.

\section{Statistical Analysis}

The study variables were as follows: peel formation (yes/no), mean IW/L, mean weight score, mean total thickness of the small bowel wall, mean thickness of the muscular layers of the small bowel wall, mean thickness of the submucosa of the small bowel wall, overall structure of the small bowel, mean inner thickness of the small bowel villi, mean outer thickness of the small bowel villi, maturity of the myenteric plexus, Hoxb5 expression (yes/no), GFAP expression (yes/no), S100 protein expression (yes/no), Hoxb5 expression level (3-point scale), GFAP expression level (3-point scale), and S100 protein expression level (3-point scale).

All statistical analyses were done using SPSSversion 15.0 for Windows (2006, SPSS Inc., Chicago, Illinois, United States). Peel formation between groups was compared using the nonparametric Kruskal-Wallis and Mann-Whitney tests. For comparisons of numerical data, we used one-way ANOVA with the Bonferroni post hoc test. $p$ values of less than 0.05 were considered significant.

\section{Results}

Survival rates after gastroschisis creation or sham procedure were $14 \%$ ( 11 fetuses $/ 80$ ) and $73 \%$ (22 fetuses $/ 30$ ), respectively. The study population included 11 gastroschisis fetuses, 22 sham fetuses, and 9 control fetuses.

\section{Peel Formation}

Peel formation was observed in 4 of the 11 gastroschisis fetuses (36\%). The macroscopic appearance of the small bowel was normal in all sham and control fetuses (-Table 2). 
Table 2 Peel assessment, morphometric analysis, and myenteric plexus maturation in the fetuses with gastroschisis, sham fetuses, and control fetuses

\begin{tabular}{|c|c|c|c|c|}
\hline & & Controls & Shams & Gastroschisis \\
\hline \multicolumn{2}{|l|}{$\mathrm{N}$} & 9 & 22 & 11 \\
\hline \multicolumn{2}{|l|}{ Peel formation } & 0 & 0 & $4(36 \%)$ \\
\hline Weight to length ratio $(\mathrm{mg} / \mathrm{cm})$ & Mean (SD) & $9.18(2.70)$ & $12.61(3.60)$ & $21.25(12.71)^{a}$ \\
\hline \multirow[t]{4}{*}{ Weight score $(\mathrm{N})$} & Weight score $=1$ & 9 & 18 & 3 \\
\hline & Weight score $=2$ & 0 & 4 & 4 \\
\hline & Weight score $=3$ & 0 & 0 & 1 \\
\hline & Weight score $=4$ & 0 & 0 & $3(27 \%)$ \\
\hline \multicolumn{2}{|l|}{ Weight score: mean (SD) } & $1(0)$ & $1.18(0.39)$ & $2.55(1.21)^{b}$ \\
\hline \multirow[t]{3}{*}{ Small bowel thickness $(\mu \mathrm{m})$} & Total: mean (SD) & $57.44(4.11)$ & $58.26(6.11)$ & $78.44(18.64)^{\mathrm{c}}$ \\
\hline & Muscular layers: mean (SD) & $22.90(2.74)$ & $23.06(4.00)$ & $30.23(8.04)^{\mathrm{d}}$ \\
\hline & Submucosa: mean (SD) & $14.45(2.50)$ & $13.84(2.63)$ & $19.98(7.82)^{\mathrm{a}}$ \\
\hline \multirow[t]{2}{*}{ Villi $(\mu \mathrm{m})$} & Inner width: mean (SD) & $12.44(1.05)$ & $11.14(1.43)$ & $12.24(3.01)$ \\
\hline & Outer width:mean (SD) & $62.81(7.28)$ & $62.02(9.32)$ & $54.96(9.46)$ \\
\hline \multirow[t]{3}{*}{ Myenteric plexus } & Mature: N (\%) & $7(78 \%)$ & $20(91 \%)$ & $0(0 \%)$ \\
\hline & Immature: N (\%) & $1(11 \%)$ & $2(9 \%)$ & $6(55 \%)$ \\
\hline & Very immature: N (\%) & $1(11 \%)$ & $0(0 \%)$ & $5(45 \%)$ \\
\hline
\end{tabular}

${ }^{\mathrm{a}} p<0.05$.

${ }^{\mathrm{b}} p<0.001$.

${ }^{c} p<0.0001$.

$\mathrm{d}_{p}<0.01$ for comparison with control and sham fetuses, respectively.

In 7 of the 11 fetuses with gastroschisis, the entire small bowel was outside the abdominal cavity, which contained the stomach and colon. Therefore, we were unable to perform a paired analysis of eviscerated and intra-abdominal small bowel. In the four fetuses with incomplete small bowel evisceration, the intra-abdominal small bowel showed no peel formation.

\section{Intestinal Weight/Intestinal Length Ratio, and Weight Score}

The mean IW/L ratio was significantly higher $(p<0.05)$ in fetuses with gastroschisis $(21.25 \mathrm{mg} / \mathrm{cm})$ than in sham fetuses $(12.61 \mathrm{mg} / \mathrm{cm})$ and control fetuses $(9.18 \mathrm{mg} / \mathrm{cm})$. In one gastroschisis fetus, extensive peel formation with severe edema and retraction precluded measurement of small-bowel length. Therefore, we defined a weight score combining IW/L values and macroscopic assessment of peel formation which allowed the evaluation of all small bowels. The mean weight score and the proportion of fetuses whose weight score was abnormal (i.e., greater than 1) were higher among the gastroschisis fetuses ( 2.55 and $73 \%$, respectively) compared with the sham fetuses (1.18 and $18 \%$, respectively; $p<0.001)$ and control fetuses (1.00 and $0 \%$, respectively; $p<0.001$ ). (-Table 2)

\section{Small Bowel Wall Thickness and Histology}

Although the overall gross anatomy of the small bowel was normal in the fetuses with gastroschisis, the subjective assessment consistently suggested thickening of the bowel wall compared with sham and control fetuses. This assessment was confirmed by the measurements of the thickness of the small bowel wall and showed that the mean total thickness of the small-bowel wall was $78.44 \mu \mathrm{m}$ in fetuses with gastroschisis compared with $58.26 \mu \mathrm{m}$ in sham fetuses and $57.44 \mu \mathrm{m}$ in control fetuses ( $p<0.0001$ for both comparisons). We also asked whether increased small bowel thickness in fetuses with gastroschisis was restricted to specific layers of the small bowel wall. To answer this question, each layer of the small bowel wall was measured for each sample. Mean thickness of both the muscular layer and the submucosa of the small-bowel wall were higher in the fetuses with gastroschisis than in the sham fetuses and control fetuses.

The overall structure and thickness of the small-bowel villi was not different between gastroschisis, sham, and control fetuses. (-Table 2 and - Fig. 2A-C)

\section{Myenteric Plexus}

The myenteric plexus were visible in the small-bowel specimens from all fetuses with gastroschisis. However, none of these fetuses exhibited normal myenteric plexus. Thus, of the gastroschisis fetuses, 55\% had an aspect similar to the immature myenteric plexus of E19-E20 fetuses and 45\% to the very immature myenteric plexus observed in E16-E18 fetuses, compared with 9 and $0 \%$ in the sham fetuses, respectively, and 11 and $11 \%$ in the control fetuses, respectively ( - Table 2 and -Figs. 2D and 3). 

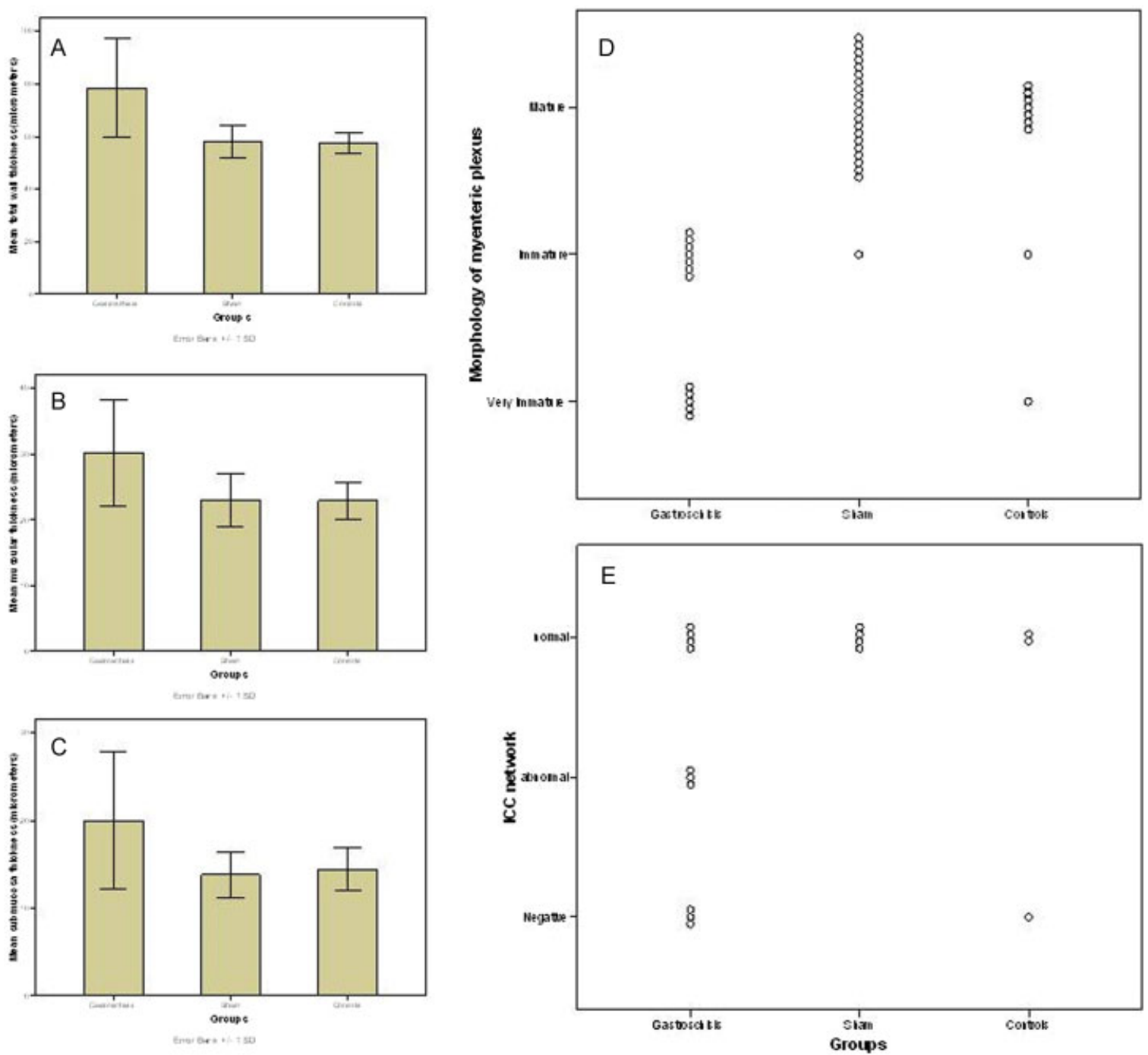

Figure 2 Panel A-C: Mean thickness of the small-bowel wall (micrometers). The error bars indicate the standard deviations. The mean total (A), muscular (B), and submucosa $(C)$ thickness of the small bowel wall were significantly higher in the fetuses with gastroschisis compared with the sham fetuses and control fetuses $(p<0.0001, p<0.01$, and $p<0.05$, respectively). Panel D: Morphology of the myenteric plexus, categorized as mature, immature, or very immature (see Methods section for definitions). Panel E: Interstitial cells of Cajal: intensity of c-kit immunostaining in the three groups.

Hoxb5, a marker for enteric neuroblasts and neuronal precursors, was expressed in myenteric plexuses in all groups ( $\sim$ Fig. 4A-C). In the gastroschisis group, $70 \%$ of fetuses displayed Hoxb5 expression in the myenteric plexus. This proportion was not different from the proportions observed in sham and control groups.

S100 or GFAP staining showed the presence of glial cells within the small-bowel myenteric plexuses in all three groups ( - Fig. 4D-H). The mean S100 expression level on the 3-point scale was 2 in all three groups (-Fig. 4D-F). In contrast, the mean GFAP expression level on the 3-point scale was higher in the gastroschisis group than in the sham and control groups ( 1.8 vs. 0.7 and 0.6 , respectively; $p<0.002$ ) ( Fig. 4G-I).

\section{Interstitial Cells of Cajal Network}

Immunostaining with antibody to c-kit identified ICCs between the circular and longitudinal muscular layers of small- bowel specimens in all three groups ( - Fig. 5). However, when compared with sham and control groups, ICCs were less abundant in eviscerated small bowel in the gastroschisis group and often exhibited weak c-kit staining or an abnormally round shape without branches (-Figs. 2E and 5).

\section{Discussion}

In this rat model of gastroschisis, we showed that extrusion of the small bowel out of the abdominal cavity during embryonic development led to peel formation, thickening of all the small bowel wall layers except villi and impaired organization of the myenteric plexus, and ICC network. These results contribute to the understanding of the intestinal dysmotility seen in neonates with gastroschisis.

Improved understanding of the mechanisms involved in small-bowel damage in gastroschisis is a prerequisite to the development of effective treatments. Most neonates with 


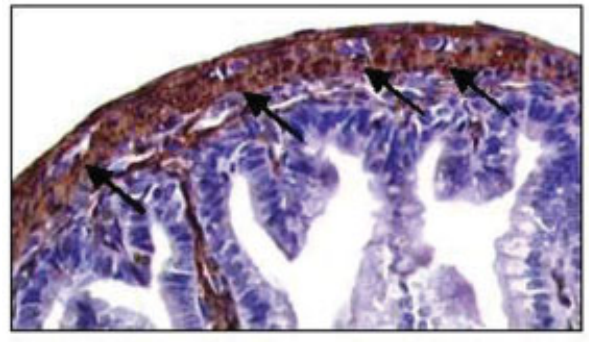

A

Control E22, x20

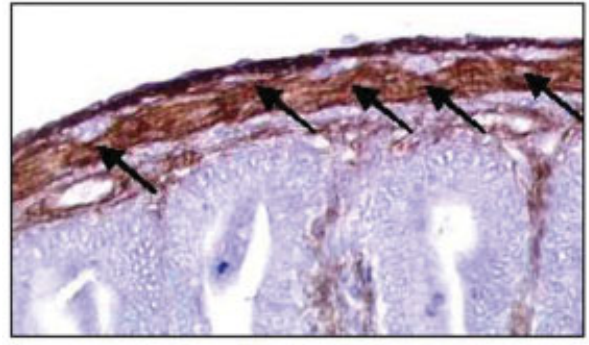

C

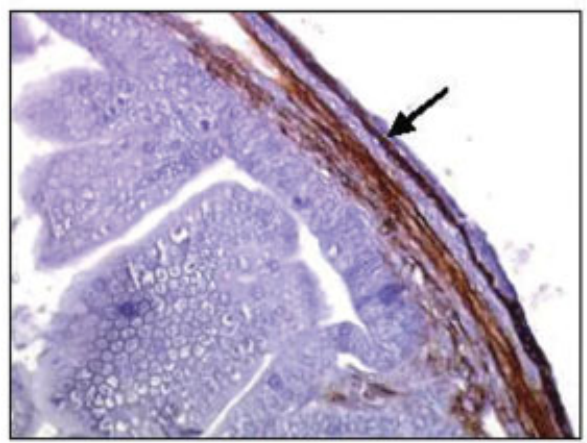

E

Gastroschisis E22, x20



Control E22, x20

B

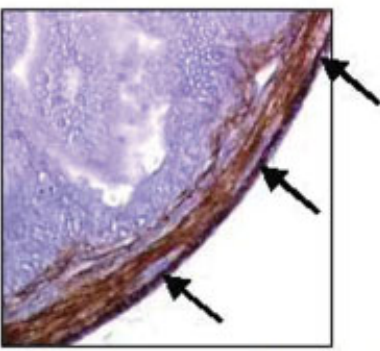

Gastroschisis E22, x20

D

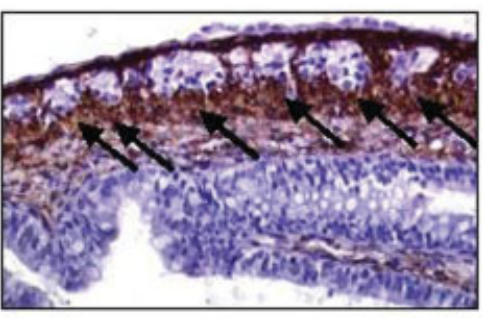

Gastroschisis E22, x20
$\mathrm{F}$

Figure 3 Small bowel on E22, magnification x20. Immunostaining with anti- $\alpha$-smooth-muscle actin antibody. Arrows indicate myenteric plexus ganglia. Panel A, B: Small bowel from control fetuses with mature myenteric plexuses. Panel C, D: Small bowel from gastroschisis fetuses with immature myenteric plexuses. Panel E, F: Small bowel from gastroschisis fetuses with very immature myenteric plexuses.

gastroschisis do not require small-bowel resection and, therefore, few human intestinal specimens are available for study; furthermore, resection is performed in humans only when the bowel exhibits severe ischemia, which confounds the results of histological studies. Therefore, investigations into the mechanisms underlying dysmotility in gastroschisis rely essentially on animal models. The animal model used in our study replicates several important features of human gastroschisis: small bowel exits the abdominal cavity through the surgical incision in the abdominal wall and is therefore exposed to the amniotic fluid; and the eviscerated bowel exhibits variable degrees of peel formation with thickening, edema, and retraction. However, the exposure to amniotic fluid was shorter than in human fetuses with gastroschisis, even after normalization for length of gestation. Furthermore, bowel ischemia related to small-bowel compression through a small defect may occur in human fetuses, as suggested both by clinical cases with overt strangulation and by experimental studies. $^{8,9}$ In our study, in contrast, we created a large abdominal defect to avoid strangulation and ischemia of the herniated bowel loops that would have compromised the analysis.

In our experimental study, we have replicated several typical features of human gastroschisis. Also, our findings agree with those of the other studies using various models of gastroschisis in rats, ${ }^{7,10-12}$ lambs, ${ }^{8}$ rabbits, ${ }^{13}$ and chicks ${ }^{14}$ : in the fetuses with gastroschisis, we found peel formation by gross examination in $36 \%$ of the cases, significantly higher values for the IW/L ratio and weight score compared with the sham and control fetuses, thickening of the serosa, muscular layers, and submucosa but normal shape and thickness of the villi.

As intestinal dysmotility is a major clinical feature of human neonates with gastroschisis, we investigated whether enteric nervous system or ICC network were affected in gastroschisis. We conducted a detailed histological and immunohistochemical study of the myenteric plexus and ICC network. We chose a qualitative method that highlights 


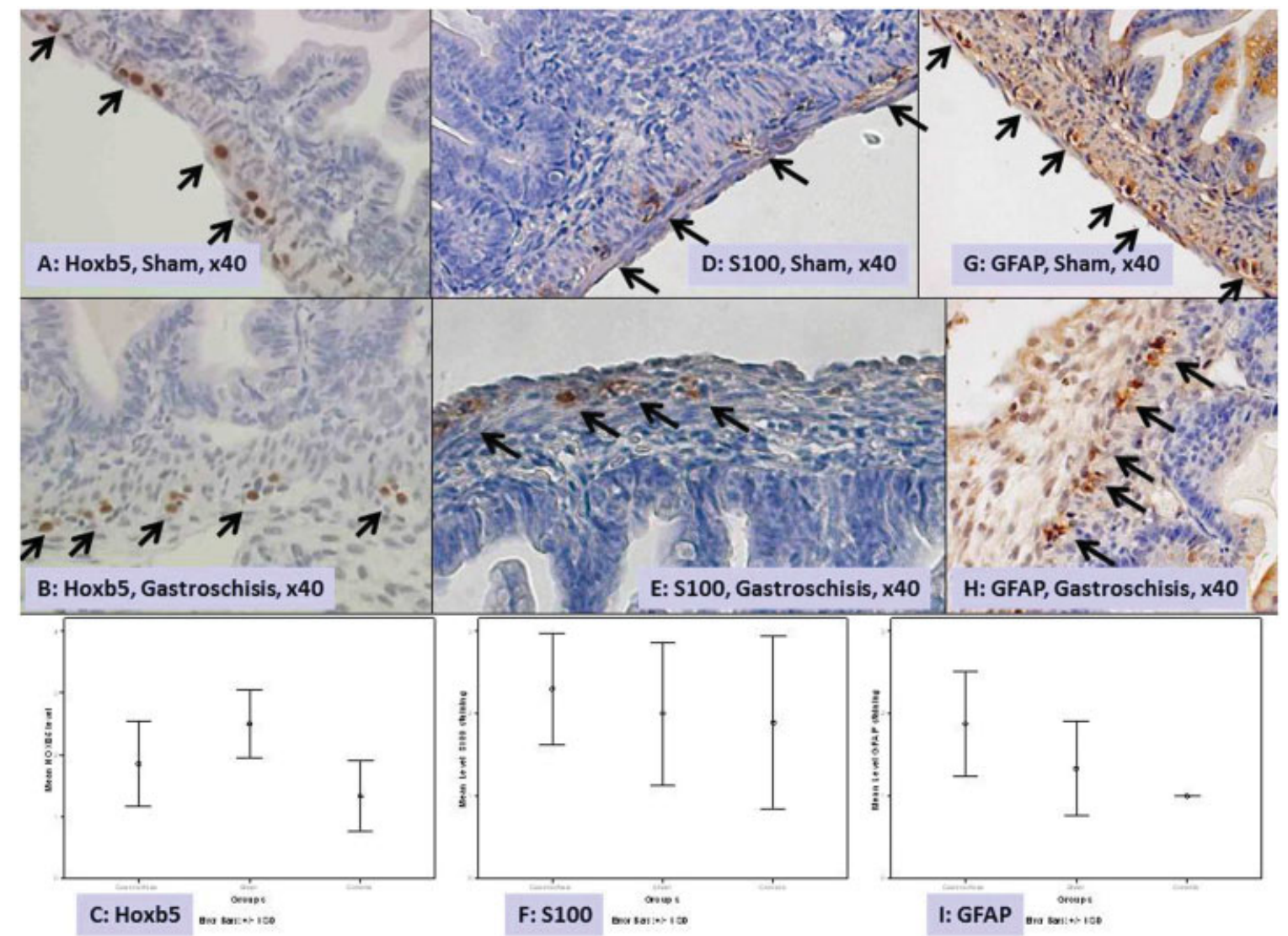

Figure 4 Panel A, B: Neuroblast precursors in small bowel in a sham fetus and a gastroschisis fetus: Hoxb5 immunostaining, magnification $\mathrm{x} 40$. Panel C: Neuroblast precursors: mean intensity of Hoxb5 immunostaining in the three groups. Panel D, E: Enteric glial cells in a sham fetus and a gastroschisis fetus: protein S100 immunostaining, magnification x40. Panel F: Enteric glial cells: mean intensity of protein S100 immunostaining in the three groups. Panel G, H: Enteric glial cells: GFAP immunostaining, magnification x40. Panel I: Enteric glial cells: mean intensity of GFAP immunostaining in the three groups. Hoxb5 and S100 (panel C and F) expression levels in the myenteric plexus of gastroschisis fetuses were not different from the expression levels observed in sham and control groups. The mean GFAP expression level (panel I) was higher in the gastroschisis group than in the sham and control groups $(p<0.002)$. The error bars indicate the standard deviations.

several dimensions of the enteric nervous system development. We performed a qualitative analysis of the myenteric plexus using morphotype comparison with specimen harvested from time-dated E16 to E22 control fetuses. We first analyzed the organization of the myenteric plexus in control fetuses during embryonic development. At E16, the myenteric plexus is organized as a continuous belt located between both smooth muscle layers on a transversal small bowel tissue section (-Fig. 1A). From E17 to E19, a partition of this continuous belt occurred and the myenteric plexus took an ovoid shape (-Fig. 1B-D). From E20 to E22, the myenteric plexus took an increasing rounded shape, with enlarged interplexus distance ( - Fig. 1E, F and - Fig. 3A, B). Similar maturation of the myenteric plexus during development has been previously described in rats and human fetuses. ${ }^{15,16}$ In our study, we compared E22 morphology of myenteric plexus from gastroschisis fetuses to control and sham fetuses, and the maturity of myenteric plexus was graded for each fetus from 1 (mature) to 3 (very immature). Although the myenteric plexus was visible in the eviscerated small bowel from gastroschisis fetuses, the structure of the plexus was consistently modified (-Fig. 3C-F). The myenteric plexus was either immature (grade $2,55 \%$ ) or very immature (grade 3 , $45 \%$ ) compared with control and sham fetuses.

Immunohistochemistry with neuronal and glial markers, as well as ICC markers, allowed us to analyze further the enteric nervous system phenotype. Our study showed glial cells stained with S100 protein and GFAP antibody and enteric neuronal precursors stained with Hoxb5 in all three groups of fetuses. Hoxb5 expression was not significantly different in the eviscerated small bowel of gastroschisis fetuses from the intra-abdominal small bowel in the sham and control fetuses suggesting that neural cells are present in gastroschisis. However, previous studies in the same rat model found decreased expression of other neuronal markers (synaptophysin, $\alpha$-internexin, and neurofilaments) in myenteric plexuses from the fetuses with gastroschisis, ${ }^{10,11}$ a finding that might indicate impaired neuronal function or phenotype.

Although expression of S100, a glial cell marker, was normal in the eviscerated bowel, the master glial-cell marker GFAP, however, was overexpressed. The glial cells ensheath the neurons within the ganglionated plexus, to insure normal function of the enteric nervous system by regulating the supply of substrate to the neurons. ${ }^{17}$ Glial cells are also 

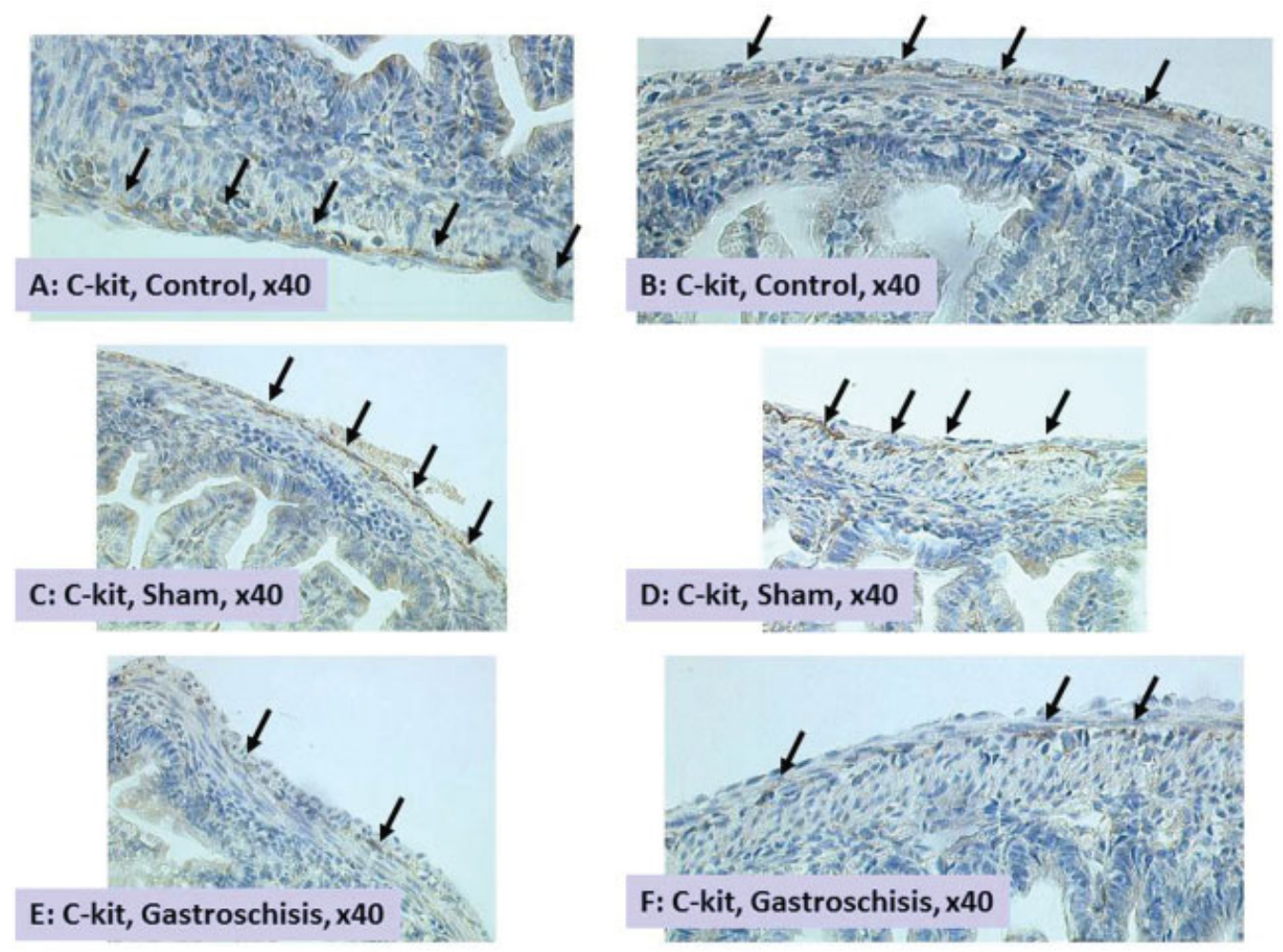

Figure 5 Interstitial cells of Cajal: c-kit immunostaining, magnification x40.

involved in scavenging neurotransmitters from the extracellular space and produce cytokines. ${ }^{17}$ Healthy intestinal specimens from patients with Crohn's disease exhibited disruption of the glial-cell network, suggesting a role for glial cells in human inflammatory bowel disease. ${ }^{18}$ Further support for such a role was obtained in a study showing that fulminant enterocolitis developed in transgenic mice devoid of enteric glia. ${ }^{19}$ We cannot determine from our data whether the GFAP overexpression in eviscerated bowel was related to increased glial cell proliferation, glial hyperplasia, increased GFAP-gene expression, or post-transcriptional modifications affecting antibody binding. However, the similar S100 expression across study groups suggested that the GFAP overexpression in the gastroschisis group was perhaps related to functional modifications in glial cell physiology. Consistent with our results, other studies have shown that GFAP expression in central nervous system glia is altered by astrocyte injury and inflammation, and GFAP overexpression in the enteric nervous system has been reported in bowel inflammation. ${ }^{17,18}$ Also, increased expression of GFAP has been observed above the defect in human with intestinal atresia, suggesting that obstruction might be a contributing factor. ${ }^{15}$ Whether GFAP overexpression reflects a protective or a detrimental glialcells response to inflammation and/or obstruction in our gastroschisis model is however unknown.

Normal intestinal motility requires normal generation and propagation of electrical slow waves throughout the smooth muscle layers. ${ }^{6}$ ICCs serve as an electrical pacemaker coupled with the smooth muscle cells via low-resistance electrical junctions. ${ }^{6}$ Small bowel lacking ICCs does not actively propa- gate slow waves and exhibits blunted responses to cholinergic and nitrergic enteric motor-neuron stimulation. ${ }^{6}$ ICCs were evaluated with specific c-kit immunostaining on small bowel specimen from all groups. In our study, ICCs in small bowel from fetuses with gastroschisis were sparse and showed weak c-kit staining or an abnormally round shape without branches, compared with the sham and control fetuses. Our findings are consistent with results from several animal models of gastroschisis. ${ }^{12,14,20,21}$ Marked intestinal dysmotility is seen in most human neonates with gastroschisis suggesting impaired development of the enteric nervous system or ICC network. Our findings support this clinical observation. Further support for this cause of delayed intestinal motility comes from a clinical case that showed delayed maturation of the ICC network and enteric neurons at birth in a specimen of normal-looking small bowel from a human neonate with gastroschisis who required resection because of bowel ischemia. ${ }^{22}$ Interestingly, examination of a second specimen taken 1 month later during surgery to restore continuity showed improved maturation of the ICC network and enteric neurons. ${ }^{22}$ Similarly, decreased expression of c-kit has been consistently observed in the proximal segment of small bowel with intestinal atresia in a large series of 23 patients ${ }^{15}$ Impairment of ICC morphology and network in our experimental gastroschisis study may thus be secondary to functional obstruction.

\section{Conclusion}

In this rat model, we were able to replicate the intestinal lesions observed in human gastroschisis, as attested by peel 
formation and parietal thickening of the eviscerated small bowel. We observed several phenotypic alterations of myenteric plexus, glial cells and ICC in the eviscerated small bowel that may be consistent with delayed maturation of the myenteric plexus, and ICC network. These features may explain the intestinal motility disorders observed in neonates with gastroschisis and give potential leads for targeted therapies.

\section{Acknowledgments}

The authors thank Prof. Alan Flake (The Center for Fetal Research, Children's Hospital of Philadelphia) for his help and critical comments; Antoneta Radu (The Center for Fetal Research, Children's Hospital of Philadelphia) and Prof. Aurore Coulomb-L'herminé (Université Pierre et Marie Curie) for their advices regarding pathology procedures; Daniel Pays (Laboratoire de chirurgie expérimentale, AP-HP-Hopital Saint-Antoine) for taking care of the animals postoperatively; MaryAnn Volpe (The Floating Hospital for Children, Tufts Medical Center, Boston) for providing the anti-Hoxb5 antibody.

This study was funded by grant CRCE No. 02003 from the Délégation Régionale de la Recherche Clinique d'Ile de France, a French public research institution.

\section{Conflict of Interest}

None

\section{References}

1 Wilson RD, Johnson MP. Congenital abdominal wall defects: an update. Fetal Diagn Ther 2004;19(5):385-398

2 Islam S. Clinical care outcomes in abdominal wall defects. Curr Opin Pediatr 2008;20(3):305-310

3 Skarsgard ED, Claydon J, Bouchard S, et al; Canadian Pediatric Surgical Network. Canadian Pediatric Surgical Network: a population-based pediatric surgery network and database for analyzing surgical birth defects. The first 100 cases of gastroschisis. J Pediatr Surg 2008;43(1):30-34, discussion 34

4 Weber TR, Au-Fliegner M, Downard CD, Fishman SJ. Abdominal wall defects. Curr Opin Pediatr 2002;14(4):491-497

5 Goyal RK, Hirano I. The enteric nervous system. N Engl J Med 1996;334(17):1106-1115

6 Ward SM, Sanders KM, Hirst GD. Role of interstitial cells of Cajal in neural control of gastrointestinal smooth muscles. Neurogastroenterol Motil 2004;16(Suppl 1):112-117
7 Correia-Pinto J, Tavares ML, Baptista MJ, Estevão-Costa J, Flake AW, Leite-Moreira AF. A new fetal rat model of gastroschisis: development and early characterization. J Pediatr Surg 2001;36(1): 213-216

8 Langer JC, Longaker MT, Crombleholme TM, et al. Etiology of intestinal damage in gastroschisis. I: Effects of amniotic fluid exposure and bowel constriction in a fetal lamb model. J Pediatr Surg 1989;24(10):992-997

9 Tibboel D, Raine P, McNee M, et al. Developmental aspects of gastroschisis. J Pediatr Surg 1986;21(10):865-869

10 Vannucchi MG, Midrio P, Flake AW, Faussone-Pellegrini MS. Neuronal differentiation and myenteric plexus organization are delayed in gastroschisis: an immunohistochemical study in a rat model. Neurosci Lett 2003;339(1):77-81

11 Vannucchi MG, Midrio P, Zardo C, Faussone-Pellegrini MS. Neurofilament formation and synaptic activity are delayed in the myenteric neurons of the rat fetus with gastroschisis. Neurosci Lett 2004;364(2):81-85

12 Midrio P, Faussone-Pellegrini MS, Vannucchi MG, Flake AW. Gastroschisis in the rat model is associated with a delayed maturation of intestinal pacemaker cells and smooth muscle cells. J Pediatr Surg 2004;39(10):1541-1547

13 Phillips JD, Kelly RE Jr, Fonkalsrud EW, Mirzayan A, Kim CS. An improved model of experimental gastroschisis in fetal rabbits. J Pediatr Surg 1991;26(7):784-787

14 Vargun R, Aktug T, Heper A, Bingol-kologlu M. Effects of intrauterine treatment on interstitial cells of Cajal in gastroschisis. J Pediatr Surg 2007;42(5):783-787

15 Khen N, Jaubert F, Sauvat F, et al; Group for the Study of Intestinal Atresia. Fetal intestinal obstruction induces alteration of enteric nervous system development in human intestinal atresia. Pediatr Res 2004;56(6):975-980

16 Fourcade L, Mousseau Y, Jaubert F, Sturtz FG. Myenteric plexus alterations downstream from a prenatal intestinal obstruction in a rat model. Neurosci Lett 2009;461(2):126-130

17 Rühl A. Glial cells in the gut. Neurogastroenterol Motil 2005; 17(6):777-790

18 Cabarrocas J, Savidge TC, Liblau RS. Role of enteric glial cells in inflammatory bowel disease. Glia 2003;41(1):81-93

19 Cornet A, Savidge TC, Cabarrocas J, et al. Enterocolitis induced by autoimmune targeting of enteric glial cells: a possible mechanism in Crohn's disease? Proc Natl Acad Sci U S A 2001;98(23): 13306-13311

20 Santos MM, Tannuri U, Maksoud JG. Alterations of enteric nerve plexus in experimental gastroschisis: is there a delay in the maturation? J Pediatr Surg 2003;38(10):1506-1511

21 Danzer E, Layne MD, Auber F, et al. Gastroschisis in mice lacking aortic carboxypeptidase-like protein is associated with a defect in neuromuscular development of the eviscerated intestine. Pediatr Res 2010;68(1):23-28

22 Midrio P, Vannucchi MG, Pieri L, Alaggio R, Faussone-Pellegrini MS. Delayed development of interstitial cells of Cajal in the ileum of a human case of gastroschisis. J Cell Mol Med 2008;12(2):471-478 